\title{
Evaluation of precision of measurement results in medical laboratory
}

\author{
A.Tumilovich 1 , T.Martynova'2, A.Chunovkina ${ }^{1}$ \\ 1 D.I. Mendeleyev Institute for Metrology (VNIIM). \\ 2 Federal State Budgetary Educational Institution of Higher Education «Academician I.P. Pavlov First \\ St. Petersburg State Medical University» of the Ministry of Healthcare of the Russian Federation (Pav- \\ lov Medical University) \\ a.a.koroleva@vniim.ru
}

\section{Summary:}

This paper analyzes an approach to evaluation of measurement uncertainty in medical laboratory. In particular, special attention is paid to precision of measurement results carried out by a medical laboratory on identical materials in precision conditions.

Keywords: medical laboratory, measurement uncertainty, certified reference material, measurement traceability, measurement precision, bias.

\section{Introduction}

Ensuring metrological traceability in the field of medical measurements aimed at realizing the possibility of comparing the measurement results obtained in different laboratories. Metrological traceability is realized through the chain of calibration performed using certified reference material and reference measurement procedure. Calibration implies calculation of associated measurement uncertainties. Knowledge of the measurement uncertainty provided by the medical laboratory is an important factor in clinical decision. The Guide to the Expression of Uncertainty of Measurement GUM [1] is a fundamental document for the calculation of measurement uncertainty. Unfortunately, uncertainty calculation methods based on measurement model are not always feasible in the field of medical measurements, therefore the document ISO/TS 20914 "Medical laboratories Practical guide for the estimation of measurement uncertainty" was developed [2]. This document follows the basic ideas of the GUM and presents a simplified method for estimating measurement uncertainty within a medical laboratory, which is based on the analysis and evaluation of measurement precision within the laboratory and information on the accuracy of reference materials/certified reference material (CRM) used by the laboratory to establish and control a bias in the measurement results.

\section{Evaluation of measurement uncertainty in medical laboratory}

According to ISO/TS 20914:2019, the uncertainty of the measurement result $u(x)$ in a medi- cal laboratory is consists of the three main components:

$$
u(x)=\sqrt{u_{\text {bias }}^{2}+u_{c a l}^{2}+u_{R w}^{2}},
$$

where $u_{\text {bias }}-$ is uncertainty of a bias value, $u_{\text {cal }}-$ uncertainty of the value assigned to an end-use calibrator, $u_{R w}-$ uncertainty estimate based on data observed under intermediate precision conditions of measurement.

In turn, the uncertainty of a bias value calculated in accordance with the formula:

$$
u_{\text {bias }}=\sqrt{S D_{\text {mean }}^{2}+u_{\text {ref }}^{2}},
$$

where $S D_{\text {mean- }}$ is standard deviation of the mean value of a measurand obtained from a repeatability study, uref - uncertainty of the value assigned to a reference material.

\section{Estimation of precision of measurement results}

This article is devoted to the assessment of the precision of measurement results ${ }^{[3]}$ based on all information available in a medical laboratory. The laboratory can assess the precision of its measurements using data from regular internal quality checks. The precision of measurements is assessed in the form of the standard deviation (SD) of the results obtained for control materials similar in their properties to the clinical samples analyzed by the laboratory. The reason for the discrepancy in the measurement results in terms of precision are: sample inhomogeneity; instrumentation; reagent and calibrator instability; reagent and calibrator lot-to-lot 
variability; fluctuations in laboratory environment; operator bias introduced by reading analogue instrument indications; more than one of the same measuring systems for the same measurand. Combining several groups into one when estimating $u_{R w}$ is performed in order to increase the accuracy of the SD estimates. The application of the Cochran test for pooling SD is discussed below.

Evaluation of the precision of measurements in a medical laboratory using the example of measuring WGB, RBC, HBL

The processing of the results was carried out jointly with the laboratory of Pavlov Medical University in which measurements were performed. Two sets of certified reference material (CRM) were analyzed for certified values of the characteristics of WGB (leukocytes), RBC (erythrocytes) and HGB (hemoglobin). Each set of CRMs consisted of two vials of certified "pathology" values (CRM1 and CRM2). In the specification for these CRMs, in addition to the certified values, their limits of the relative error for a confidence level of $0.95 \%$ were indicated. Also measurement results for control material (CM), consisting of the values "pathology" for the investigated characteristics of WGB, RBC, HGB were obtained.

In order to evaluate the precision for each characteristic of CRMs and CM, 10 measurements were carried out on the same measuring instrument under the same precision conditions. Thus, the medical laboratory received 10 values of characteristics related to "pathology" from the 1 st set, 10 values of characteristics related to "pathology" from the 2nd set and 10 values of characteristics related to the measurement of CM. Further, according to ISO/TS 20914:2019, the SD was estimated for each characteristic from each set. To characterize HGB, instead of the $S D$, the range was estimated, since distribution of measurement results differs from the normal distribution.

Cochran's criterion was used to analyze the homogeneity of the variance of the measurement the characteristics of WBC and RBC, in accordance with formula (3):

$$
\frac{S D_{\max }^{2}}{\sum S D_{i}^{2}} \leq G_{0,95}(3,10)
$$

where $S D_{\max }$ - is the largest value of the variance of each characteristic, $G_{0,95}(3,10)$ - is the critical value of the Cochran criterion for the significance level 5\%, equal to 0.617 .
The data on the estimated variances of characteristics and the analysis of homogeneity by the Cochran test are presented in Table 1:

Tab. 1: Analysis of homogeneity

\begin{tabular}{|c|c|c|}
\hline MD & $\begin{array}{c}\text { WGB, } \\
10^{9} / 1\end{array}$ & $\begin{array}{c}\text { RBC } \\
10^{12} / 1\end{array}$ \\
\hline SD (CRM1) & 0.079 & 0.034 \\
\hline SD (CRM2) & 0.089 & 0.026 \\
\hline SD (CM) & 0.075 & 0.046 \\
\hline Cochran criterion & $0.032<0.617$ & $0.020<0.617$ \\
\hline$S D_{\sum}=\sqrt{\frac{\sum S D_{i}^{2}}{3}}$ & 0.081 & 0.036 \\
\hline
\end{tabular}

The range of measurement results of HGB is 2 $\mathrm{g} / \mathrm{l}$ for RCMs and $4 \mathrm{~g} / \mathrm{L}$ for $\mathrm{CM}$ that corresponds to $2 \%$ and $6 \%$ accordingly.

\section{Conclusions}

According to the obtained results, the hypothesis about the homogeneity of the variances of measurement results of WGB, RBC and HGB is confirmed. Therefore, for each of the analyzed characteristics, the pooled variance $\left(\mathrm{SD}_{\Sigma}\right)$ can be attributed to the measurement results of WBC, RBC, obtained in further measurements in this medical laboratory. The article describes the procedure for evaluating the SD of the precision of measurements in a medical laboratory based on the pooled estimate of variance. The obtained estimate can be used in carrying out the internal laboratory control of the measurement precision and in the estimation of the measurement uncertainty. In the extended paper the method of combining information on the variance of precision based on the Bayesian approach will be presented and compared the one considered.

\section{References}

[1] JCGM 100:2008 Evaluation of measurement data - Guide to the expression of uncertainty in measurement (GUM);

[2] ISO/TS 20914 «Medical laboratories - Practical guidance for the estimation of measurement uncertainty»;

[3] JCJM 200:2008 International vocabulary of metrology - Basic and general concepts and associated terms (VIM);

[4] ISO 5725-2:2019 Accuracy (trueness and precision) of measurement methods and results Part 2: Basic method for the determination of repeatability and reproducibility of a standard measurement method. 